\title{
Damping of Elastically Supported Element in a Vacuum Tube
}

\author{
S. Levy, E. V. Hobbs, W. D. Kroll, and L. Mordfin
}

\begin{abstract}
It is shown that it is possible to damp the motion of an element in a vacuum tube by making the tube part of a two-degree-of-freedom system. The equations of motion of this system are presented. The optimum values of the parameters are determined on SEAC, the National Bureau of Standards electronic automatic computer, for a particular set of design requirements. In carrying out this computation, SEAC is used not only to compute the displacements of the system for given values of the parameters, but also to choose that set of parameters for which the performance is optimum. A description is given of a tube mount built in accordance with the computations and of the degree of damping achieved.
\end{abstract}

\section{Introduction}

In the development of the Ramberg vacuum-tube accelerometer, ${ }^{1}$ the problem arose of eliminating excessive electrical output in the neighborhood of the resonant frequency. One solution of this problem was to include an electrical filter in the output of the accelerometer to electrically remove the effects of resonance of the mechanical system. As long as the resonant amplitude was in the linear range, such a filter was adequate. In some applications, however, large resonant amplitudes, beyond the range of linearity between mechanical displacement and electrical output, might be present, and for these it was considered desirable to damp the mechanical vibration directly.

The damping system described herein was especially designed to give good damping for the Ramberg vacuum-tube accelerometer. The design requirements were those for an accelerometer, namely, constant displacement amplitude and phase shift changing linearly with frequency, for frequencies from zero up to the natural frequency. The methods presented for achieving these design requirements are general and can be used where other types of damping are required

\section{Mechanical System}

A diagrammatic sketch of the mechanical system used to damp response of an elastically supported element in a vacuum tube is shown in figure 1 (a).

A difficulty in damping the motion of elements of a vacuum tube by dampers attached to the tube base is that the elements are usually so light that their motion causes negligibly small motion of the tube base. To increase the motion of the tube base, and thus the absorption of energy from a particular vibrating element, the auxiliary mass $m$ is used in the design shown in figure 1 (a). This mass is supported on a spring $k$ so chosen that the natural frequency of the mass, with the tube base clamped, is the same as the natural frequency of the element to

1٪Walter Ramberg, Vacuum tube acceleration pickup, J. Research NBS 37, 391 (December 1946) RP1754. The measurement of acceleration with a vacuum tube, Trans. Am. Inst. Elec. Engrs. 66 (1947). Samuel Levy, Recent developments in the Ramberg vacuum tube accelerometer, Proc. Soc. Exp. Stress Analysis. IX, No. 1 (1951)

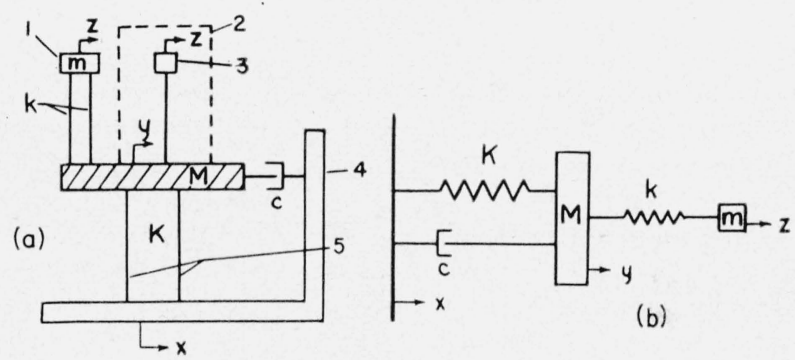

Figure 1. a, Schematic diagram of mechanical arrangement for damping response of an elastically supported element in a vacuum tube; b, equivalent two-degree-of-freedom mechanical. system.

1. Auxiliary external mass elastically supported on cantilever springs; 2 , tube envelope; 3 , elastically supported element; 4 , case; 5 , cantilever springs mounting tube in case.

be damped. Under these circumstances, the response of the tube element and the mass, $m$, to motion of the tube base is the same. The mass $m$ is large enough to force appreciable motion of the tube base, with a resultant damping of the motion of both $m$ and the tube element by damper $c$.

The springs $K$ support the tube from the outside case.

\section{Equations of Motion}

For purposes of analysis, the mechanical system of figure 1 (a) is idealized to that of figure 1 (b). The displacement $(z-y)$ of mass $m$ (and therefore of the tube element) to motions $x$ of the case will now be determined.

Writing the equations of equilibrium for masses $m$ and $M$ gives

$$
m\left(d^{2} z / d t^{2}\right)+k(z-y)=0 .
$$

$M\left(d^{2} y / d t^{2}\right)+K(y-x)+k(y-z)+c(d y / d t)-c(d x / d t)=0$.

These equations are solved on the assumption that the disturbance at the case is of constant amplitude $a$ and radian frequency $\omega$

$$
x=a \sin \omega t .
$$


The following substitutions are made:

$$
k / m=\theta^{2} ; \quad K / M=\Omega^{2} ; \quad c / M=\gamma ; \quad m / M=\rho .
$$

After solving eq (1) and (2), it is found that the acceleration of the mass $m$ is

$$
k(z-y) / m=\theta^{2}(z-y)=\mathrm{a} \omega^{2}(A \sin \omega t+B \cos \omega t),
$$

where

$$
\begin{aligned}
& A=\frac{\theta^{2} \Omega^{2}\left(\omega^{4}-\omega^{2} \theta^{2}-\omega^{2} \Omega^{2}-\omega^{2} \rho \theta^{2}+\Omega^{2} \theta^{2}\right)-\theta^{2} \omega^{2} \gamma^{2}\left(\omega^{2}-\theta^{2}\right)}{\omega^{2} \gamma^{2}\left(\omega^{2}-\theta^{2}\right)^{2}+\left(\omega^{4}-\omega^{2} \theta^{2}-\omega^{2} \Omega^{2}-\omega^{2} \rho \theta^{2}+\Omega^{2} \theta^{2}\right)^{2}}, \\
& B=\frac{\omega \gamma \theta^{2} \Omega^{2}\left(\omega^{2}-\theta^{2}\right)+\gamma \omega \theta^{2}\left(\omega^{4}-\omega^{2} \theta^{2}-\omega^{2} \Omega^{2}-\omega^{2} \rho \theta^{2}+\Omega^{2} \theta^{2}\right) .}{\omega^{2} \gamma^{2}\left(\omega^{2}-\theta^{2}\right)^{2}+\left(\omega^{4}-\omega^{2} \theta^{2}-\omega^{2} \Omega^{2}-\omega^{2} \rho \theta^{2}+\Omega^{2} \theta^{2}\right)^{2}} .
\end{aligned}
$$

The ratio of the maximum acceleration $\theta^{2}(z-y)_{\max }$ of the mass $m$ to the maximum acceleration $a \omega^{2}$ of the case is

$$
\text { Response ratio, } R=\sqrt{A^{2}+B^{2}} \text {. }
$$

The phase shift of the amplitude $(z-y)$ with respect to the motion $x$ of the base is seen from eq (5) to be

$$
\phi=\tan ^{-1}(B / A) .
$$

\section{Application of SEAC}

It is desired to determine the values of $\rho=m / M$, $\Omega=K / M$, and $\gamma=c / M$ for which the response ratio, eq (6), has a constant value near unity for values of the driving frequency $\omega$ between 0 and $\theta$. This was done by preparing a code for SEAC, ${ }^{2}$ which required it to carry out automatically the following successive steps:

(1) For values of $\omega / \theta=0.1, \Omega / \theta=0.1, \gamma / \theta=1.0$, and $\rho=0.6$ compute the values of $A$ and $B$, eq (5), and from them the response ratio, $R$, eq (6). Store $R$ temporarily.

(2) Increase the value of $\omega / \theta$ successively to 0.3 , $0.5,0.7$, and 0.9 , repeating step (1) for each new value.

(3) Determine the maximum, $R_{\max }$, of $R_{0 \cdot 1}, R_{0 \cdot 3}$, . . $R_{0.9}$ and store temporarily.

(4) Compute a criterion $C$ of flat response from. $C=R_{0.1}+0.3 R_{0.3}+0.1 R_{0.5}+.03 R_{\max }-1.43$ and store temporarily.

(5) Increase $\Omega / \theta$ in steps of 0.1 from 0.1 to 1.6 , $\gamma / \theta$ in steps of 0.1 from 1.0 to 2.0 , and $\rho$ in steps of 0.2 from 0.6 to 2.0 , and repeat steps (1) to (4) saving the results for the best five cases (those where the absolute value of $C$ is smallest).

(6) Print the results for the five best cases.

The best values were

$$
\Omega / \theta=0.1, \quad \rho=1.8, \quad \text { and } \gamma / \theta=1.9 .
$$

The curve of response ratio versus frequency ratio corresponding to these values is shown in figure 2 .

2 SEAC, the National Bureau of Standards Eastern Automatic Computer, NBS Technical News Bulletin 34, p. 9, (Sept. 1950).

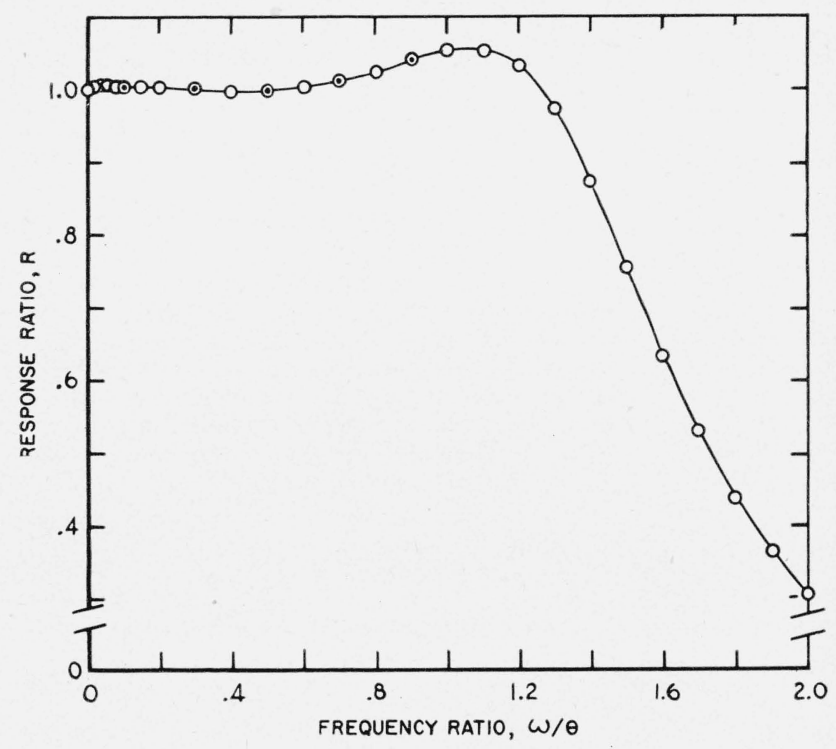

Figure 2. Response curve for optimum values of design variables.

$(\gamma / \theta=1.9 ; \rho=1.8 ; \Omega / \theta=0.1)$

- Computed on SEAC; $\bigcirc$, computed by hand.

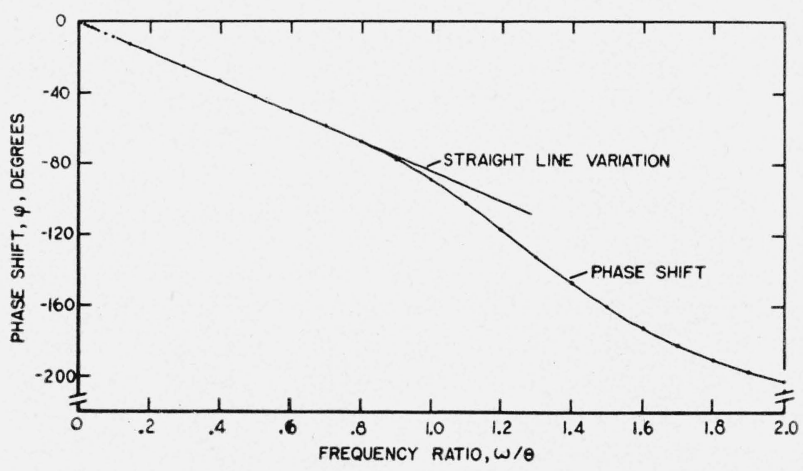

Figure 3. Phase shift for optimum values of design variables. $(\gamma / \theta=1.9 ; \rho=1.8 ; \Omega / \theta=0.1)$

The response ratio is within 1 percent of 1.00 for values of $\omega / \theta$ less than 0.67 . It is within 6 percent of 1.00 for $\omega / \theta$ less than 1.34. The phase shift for this case was computed from eq (7) and is plotted in figure 3. The departure from a straight line is negligible for $\omega / \theta$ less than 0.75 . It should be noted that the additional points computed by hand for figure 2 fit in perfectly with those computed on SEAC

An important operation of SEAC for this problem was that it not only computed the responses but also, with the aid of the criterion $C$, chose the parameters for which the response curve was flattest. In this way, the plotting by hand and the visual comparison which might otherwise have been needed were avoided.

In the coding of this problem for SEAC, no effort was made to include the linearity of the phase shift as part of the criterion $C$. This could have been done, with only minor changes in the coding, if it had been found necessary. 

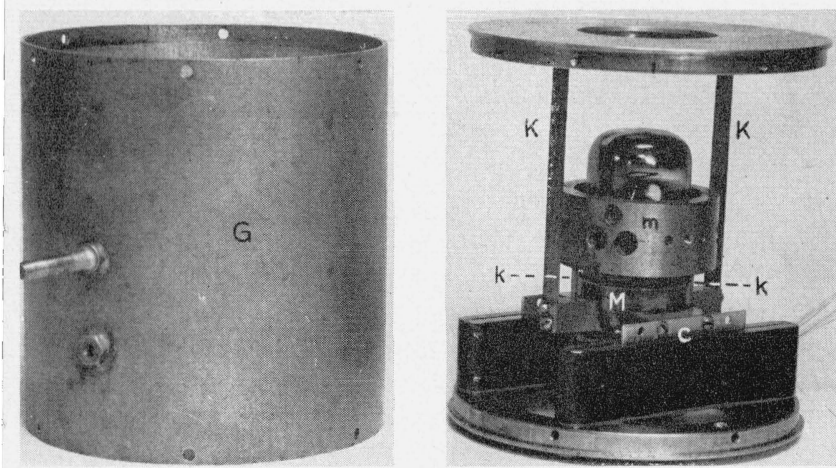

FIgURE 4. Ramberg vacuum tube accelerometer as part of a two-degree-of-freedom system having optimum parameters for damping resonant amplitudes and giving the accelerometer a flat frequency-response curve.

\section{Damper Design}

A damper designed as indicated in eq (8) was constructed and is shown in figure 4 with the outer case $G$ removed. The masses $m$ and $M$, the springs $k$ and $K$, and the damper $c$ are labeled on the figure. The element of the Ramberg vacuum-tube accelerometer for which damping was desired had a natural frequency $\theta=2 \pi(161) \mathrm{rad} / \mathrm{sec}$. The tube, together with the necessary base attachments, had a mass $M=0.000285 \mathrm{lb}-\mathrm{sec}^{2} / \mathrm{in}$. Using the optimum parameters given in eq (8), the elements in figure 1 were designed to have the following values:

$m=\rho M=1.8(0.000285)=0.000513 \mathrm{lb}-\mathrm{sec}^{2} /$ in.

$k=m \theta^{2}=(0.000513) 4 \pi^{2}(161)^{2}=526 \mathrm{lb} /$ in.

$c=\gamma M=1.9 \theta M=1.9(2 \pi)(161)(0.000285)=$

$0.548 \mathrm{lb}-\mathrm{sec} / \mathrm{in}$.

$K=M \Omega^{2}=M(0.1 \theta)^{2}=(0.000285) 4 \pi^{2}(16.1)^{2}=$

$2.92 \mathrm{lb} / \mathrm{in}$.

\section{Electrical Output at Natural Frequencyl}

To determine whether the damper was successfu in limiting electrical output at the natural frequencythe tube was subjected to a sudden change in accel eration. Such a change in acceleration should excite vibration at the natural frequency. Since the electrical output of the Ramberg vacuum-tube accelerometer is a direct measure of the displacement of the elastically supported element, the effect of the case on the vibration of the tube element could be conveniently determined by recording the accelerometer output. The desired sudden change in acceleration was obtained by mounting the tube at the end of a cantilever beam $A$, figure 5 , whose tip was deflected sideways by a force, $W$, of several times the weight of the mounted tube, $T$. Cutting the support wire, $S$, caused the desired sudden change in acceleration.

The results of this test are shown in figure 6 . It is evident from comparison of figures 6 (a), with the

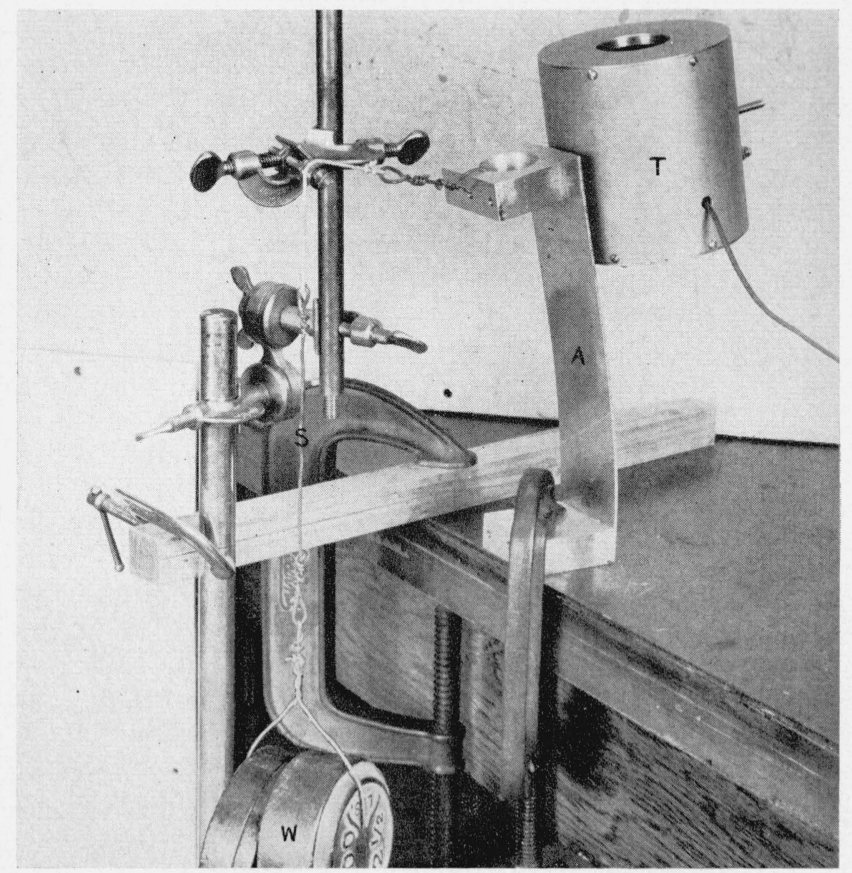

FIgure 5. Test set-up for determining the degree of damping of natural frequency achieved by making tube part of a twodegree-of-freedom system.

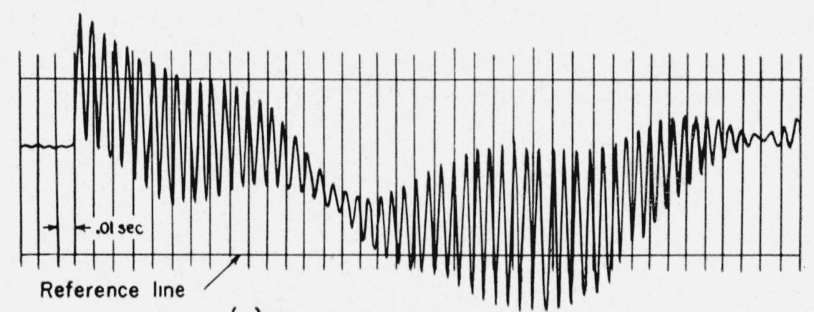

(a) Record, damper locked

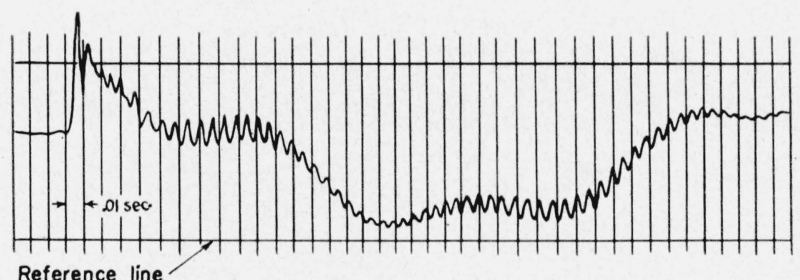

(b) Record, damper unlocked

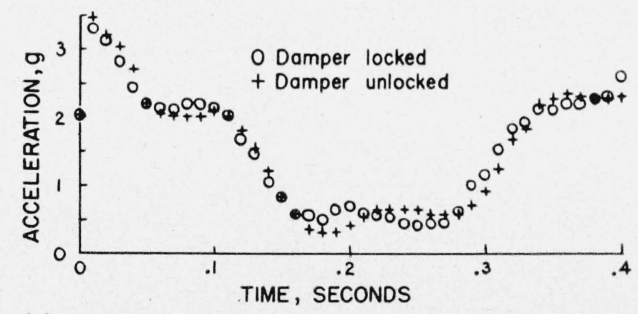

(C) Accelerations obtained by fairing curve through natural frequency response of above records

FIGURE 6. Records of acceleration obtained in tests to determine effectiveness of damper in reducing response at $161 \mathrm{c} / \mathrm{s}$ natural frequency of tube. 
damper locked to prevent relative motion between the tube and the cantilever tip, and 6 (b) with the damper unlocked that a marked reduction in natural frequency electrical output results from use of the damper. In figure 6 (c) it is seen that the acceleration-time history obtained by fairing a curve through the natural frequency response in figures 6 (a) and 6 (b) gives nearly identical results. This is expected since none of the output in figure 6 (a) exceeds the linear range of about $6 \mathrm{~g}$ for the $161 \mathrm{c} / \mathrm{s}$ model of the Ramberg vacuum-tube accelerometer.

\section{Accelerometer Performance}

Preliminary tests have been completed to determine the extent to which the performance of the accelerometer is in agreement with the computed curves, figures 2 and 3 . In these tests, the accelerometer was driven by an electromechanical shaker at frequencies from 10 to $200 \mathrm{c} / \mathrm{s}$, and the output of the accelerometer was viewed on an oscilloscope. It was observed that large amplitude electrical output at the natural frequency was present. This has been tentatively ascribed to nonlinearity. Other damping fluids and dash-pot designs will be considered in an effort to eliminate $t^{\prime}$ is difficulty.

\section{Conclusions}

The following conclusions can be drawn from this investigation:

(1) The vibration of an element in a vacuum tube due to external disturbances can be damped by making the tube a part of a two-degree-of-freedom mechanical system having suitable constants.
(2) The National Bureau of Standards electronic automatic computing machine, SEAC, is an excellent means for computing the displacements of dynamic systems, particularly where it is desired to compute the displacements for many different values of the mechanical parameters.

(3) SEAC can be conveniently used as a substitute for hand plotting of results and visual inspection of curves in selecting optimum parameters of a system by having SEAC compute, in addition to a solution of the problem, a criterion of quality and having it select the solution for which the criterion is best.

(4) Further work is indicated to investigate the cause of and to eliminate the large amplitude electrical output of the accelerometer when driven at its natural frequency.

The authors acknowledge the assistance of members of the Engineering Mechanics Section of the National Bureau of Standards, particularly that of the late A. E. McPherson. They also take this opportunity to acknowledge the major contribution of Ida Rhodes of the Bureau's Computation Section in coding the dynamic analysis for SEAC and in helping formulate the analysis so that SEAC could be used to best advantage.

The work was carried out in the Engineering Mechanics Section, National Bureau of Standards, under the sponsorship of the Office of Basic Instrumentation with the financial assistance of the Office of Naval Research, Office of Air Research and Atomic Energy Commission.

Washington, July 17, 1952. 